\title{
A systematic literature review of existing tools used to assess medication adherence in connective tissue diseases: the state of the art for the future development of co-designed measurement tools
}

\author{
S. Pirri' ${ }^{1}$, R. Talarico ${ }^{2}$, D. Marinello ${ }^{2}$, G. Turchetti' ${ }^{1}$, M. Mosca ${ }^{2}$ \\ 'Institute of Management, Scuola Superiore Sant'Anna, Pisa; \\ ${ }^{2}$ Rheumatology Unit, Azienda Ospedaliero Universitaria Pisana, Pisa
}

\begin{abstract}
SUMMARY
Lack of medication adherence is frequent in chronic connective tissue diseases and is associated with poorer health outcomes, low quality of life and economic loss.

This research is based on a systematic literature search and aims to identify the surveys and tools used for the assessment of medication adherence in patients with connective tissue diseases (CTDs) and in particular the tools co-designed with patients.

A systematic literature review was performed in PubMed and Embase databases searching for studies concerning the application of surveys or tools designed for medication adherence assessment. A specific analysis was also performed to identify which of these existing tools were developed in co-design with patients affected by CTDs. 1958 references were identified, and 31 studies were finally included. Systemic lupus erythematosus was the most investigated disease, followed by the Behçet's disease. The tools used to assess adherence in CTDs were, in most cases, valid and useful. However, the results showed a certain degree of heterogeneity among the studies and the medication adherence assessment and measurement tools adopted, which were mostly based on selfreported questionnaire. No co-designed tools with patients were found.

Low- and non-adherence were explored in some CTDs with valid and useful tools, while other CTDs still need to be assessed. Therefore, more efforts should be made to better understand the specific reasons for the low- and non-adherence in CTDs patients.
\end{abstract}

Key words: Literature review, medication adherence, connective tissue diseases, score, instruments, outcomes, co-design, questionnaires.

Reumatismo, 2021; 73 (3): 135-146

\section{INTRODUCTION}

A dherence has been defined as the 'extent to which a person's behavior (in terms of taking medications, following diets, or executing other lifestyle changes) corresponds with agreed recommendations from a health care provider' (1).

Depending on the types of disease, many factors and variables may affect the complexity of managing a treatment regimen. Self-care instructions can range from relatively simple treatment plans, such as taking a medication daily for preventive purposes, to remarkably complex daily plans, like taking multiple medications, monitoring symptoms or side effects, or making deep lifestyle changes like diets, daily exercise and other behavioral adjustments (2).

The lack of medication adherence leads to poorer health outcomes for the patients, which affect their quality of life, cause economic loss for the healthcare system and trigger uncertainty for the healthcare prescribers in dealing with the disease treatment $(3,4)$.

Despite the recognized harmful impact of non-adherence, the rate of patients who do not comply with their treatment regimens is still around $50 \%$ in most chronic conditions $(5,6)$.
Corresponding author: Salvatore Pirri Institute of Management, Scuola Superiore Sant'Anna Via Santa Cecilia, 3 - 56127 Pisa E-mail: s.pirri@santannapisa.it 
Over the last few decades, many approaches to measuring the magnitude of this complex issue were explored. However, healthcare professionals and researchers still have very little guidance on how to select the most suitable measurements to understand the mechanisms leading to non-adherence to treatment (7).

Undirect measurements like surveys and self-reported assessments are the most common methods used to rate adherence (8). Self-reported tools have the advantage of being practical, flexible, low cost and able to receive quick feedbacks thanks to their ease-of-use, as for example they can be shared through online assessments, paper questionnaires, interviews, etc. This has contributed to making them popular in many clinical settings (9).

However, in addition to the presence of recall biases, a big drawback of undirected measurements is that patients tend not to be completely reliable on reporting the real adherence rate (social desirability bias). This often happens because patients are concerned about generating negative opinions about them, so they tend to give answers that are considered more socially acceptable and in accordance with the expectations of health professionals (10).

Since the 1970s, collaboration with endusers from the very early stages of the design of a new service or a new product has been considered a consolidated approach in many industries (11). Despite this wellknown strategy, the actual application of this kind of marketing approach is still little known in many healthcare domains (12). Nevertheless, a recent study showed how patient's experience data are positively associated with patient safety and clinical effectiveness (13). It is clear that patients' involvement in co-designing healthrelated tools and services may have a concrete positive impact, especially when all the stakeholders involved are mutually engaged. This approach could be extremely valuable in identifying the barriers and limitations causing low- and no-adherence in patients (14). This is particularly true in autoimmune rheumatic diseases and connective tissue and musculoskeletal diseases, such as systemic lupus erythematosus (SLE), Sjögren's syndrome or Ehlers-Danlos syndrome, in which the lack of medication adherence is also an issue $(15,16)$.

Connective tissue and musculoskeletal diseases (CTDs) encompass a considerable number of diseases and syndromes, including rare connective tissue diseases (systemic sclerosis, mixed connective tissue disease, inflammatory idiopathic myopathies, undifferentiated connective tissue diseases, anti-phospholipid syndrome), and complex connective tissue diseases (systemic lupus erythematosus, Sjögren's syndrome).

The extensive variability of the clinical pictures among different patients and also in the

Table I - MeSH terms search query for PubMed and EMBASE.

\begin{tabular}{|c|c|}
\hline $\begin{array}{l}\text { ("Treatment Adherence and Compliance"[Mesh] OR "Medication Adherence"[Mesh] OR "Patient Compliance"[Mesh]) } \\
\text { AND ("Mixed Connective Tissue Disease"[Mesh] OR "Connective Tissue Diseases"[Mesh] OR "Undifferentiated } \\
\text { Connective Tissue Diseases"[Mesh] OR "Lupus Erythematosus, Systemic"[Mesh] OR "Sjogren's Syndrome"[Mesh] OR } \\
\text { "Ehlers-Danlos Syndrome"[Mesh] OR "Polychondritis, Relapsing"[Mesh] OR "Antiphospholipid Syndrome"[Mesh] OR } \\
\text { "Immunoglobulin G4-Related Disease"[Mesh] OR "Scleroderma, Systemic"[Mesh] OR "Behçet's Disease"[Mesh] OR } \\
\text { "Scleroderma, Diffuse"[Mesh] OR "Polymyositis"[Mesh] OR "Dermatomyositis"[Mesh]) NOT ("Arthritis, Juvenile"[Mesh] } \\
\text { OR "Arthritis, Rheumatoid"[Mesh] OR "Rheumatoid Arthritis, Systemic Juvenile" [Supplementary Concept]) }\end{array}$ & $\begin{array}{l}\text { PubMed search } \\
\text { query: } \\
946 \text { articles }\end{array}$ \\
\hline $\begin{array}{l}\text { 'treatment adherence and compliance'/exp AND ('mixed connective tissue disease'/exp OR 'mixed connective tissue } \\
\text { disease' OR 'connective tissue diseases'/exp OR 'connective tissue diseases' OR 'undifferentiated connective tissue } \\
\text { diseases'/exp OR 'undifferentiated connective tissue diseases' OR 'lupus erythematosus, systemic'/exp OR 'lupus } \\
\text { erythematosus, systemic' OR 'sjogren/s syndrome'/exp OR 'sjogren syndrome' OR 'ehlers-danlos syndrome'/exp } \\
\text { OR 'ehlers-danlos syndrome' OR 'polychondritis, relapsing'/exp OR 'polychondritis, relapsing' OR 'antiphospholipid } \\
\text { syndrome'/exp OR 'behçet disease'/exp OR 'antiphospholipid syndrome' OR 'immunoglobulin g4-related disease'/ } \\
\text { exp OR 'immunoglobulin g4-related disease' OR 'scleroderma, systemic'/exp OR 'scleroderma, systemic' OR } \\
\text { 'scleroderma, diffuse'/exp OR 'scleroderma, diffuse' OR 'polymyositis'/exp OR 'polymyositis' OR 'dermatomyositis'/ } \\
\text { exp OR 'dermatomyositis') NOT ('arthritis, juvenile'/exp OR 'arthritis, juvenile' OR 'arthritis, rheumatoid'/exp OR 'arthritis, } \\
\text { rheumatoid' OR 'rheumatoid arthritis, systemic juvenile') }\end{array}$ & $\begin{array}{l}\text { EMBASE search } \\
\text { query: } \\
1012 \text { articles }\end{array}$ \\
\hline
\end{tabular}


same patients over time represents a huge clinical challenge in the management of CTDs. This variability is related to different factors, such as the clinical manifestations of the diseases, the fluctuation between remission and exacerbation over time, the coexistence of manifestations related to irreversible damage, the occurrence of comorbidities and drug toxicities and the patient's related quality of life. In order to overcome this significant complexity, an interdisciplinary approach is absolutely crucial for the diagnosis and management of CTDs.

Such heterogeneous group of rare and complex connective conditions, which share some common immunopathogenic mechanisms, makes it complex for healthcare professionals to deal effectively with the treatment plan.

The main objectives of this systematic literature search were:

1) to identify, in the published literature, tools developed to measure and/or assess medication adherence in CTDs patients;

2) to explore whether the existing tools used to measure and/or assess medication adherence were developed in codesign with CTDs patients.

\section{MATERIALS AND METHODS}

We performed a systematic literature review in accordance with the Preferred Reporting Items for Systematic Reviews and

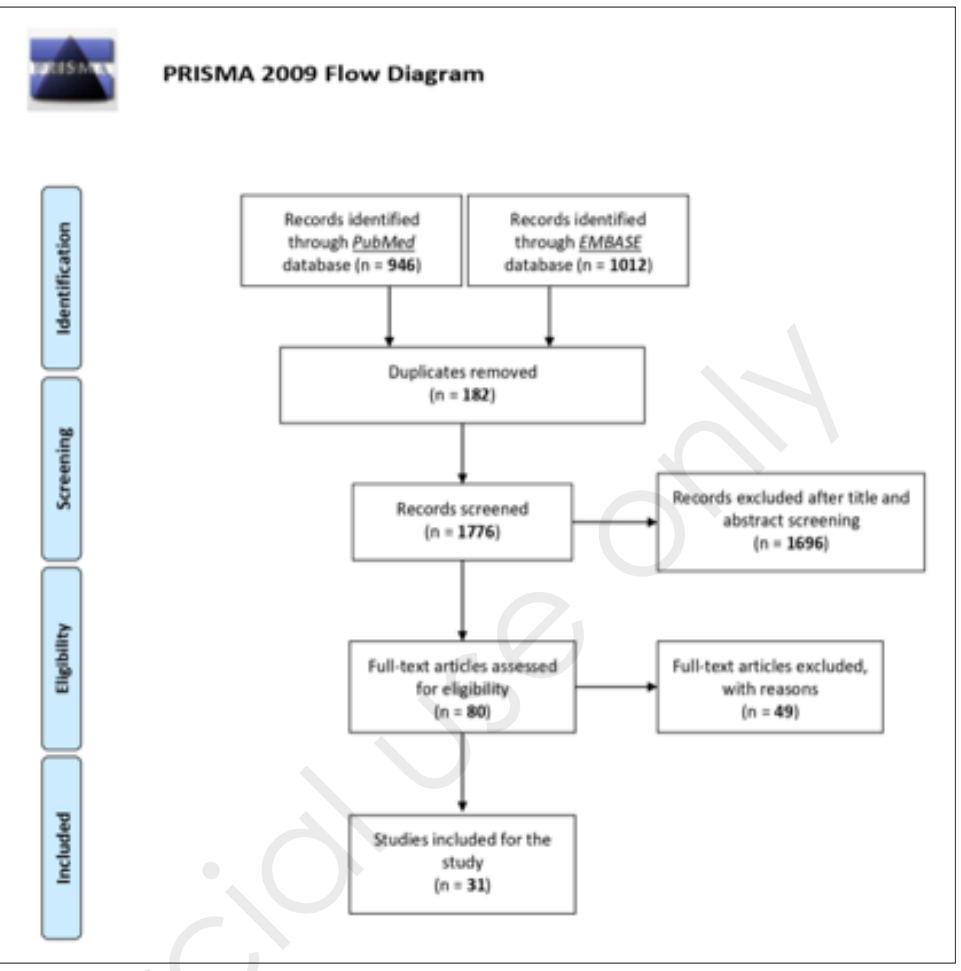

Figure 1 - PRISMA flow-chart.

Meta-Analyses (PRISMA) guideline (17). The literature search was carried out in PubMed and EMBASE academic databases without any limitation in terms of dates. All the available publications until the $12^{\text {th }}$ May 2020 were considered. The following $\mathrm{MeSH}$ terms were employed as search queries (Table I).

Table II - Selection criteria for studies eligibility.

Inclusion criteria:

- Observational and experimental studies that report data on the adherence through surveys evaluating adherence, persistence, discontinuation of CTDs patients;

- Studies including adult patients ( $\geq 18$ years old);

- Language: English;

- Peer-reviewed and full-text article available;

- CTD diseases considered were mixed connective tissue disease, connective tissue diseases, undifferentiated connective tissue diseases, systemic lupus erythematosus, Sjögren's syndrome, Ehlers-Danlos syndrome, Behçet's disease, relapsing polychondritis, Antiphospholipid syndrome, IgG4-related disease, systemic sclerosis, polymyositis, dermatomyositis.

Exclusion criteria:

- Studies that did not evaluate adherence, persistence, discontinuation in a clear statement;

- Studies that did not consider surveys or co-design methods with patients;

- Studies performed on other diseases than CTDs;

- Studies that did not employ surveys, chart medical records or registries;

- Studies with undefined data source;

- Papers not available in English. 
To achieve the study objectives and ensure an accurate result a blind check of the articles retrieved in the literature was performed independently by two authors, SP and RT, according to the inclusion and exclusion criteria shown in Table II. Disagreement between the two reviews was resolved by consensus with the other authors.

\section{RESULTS}

The results of the PRISMA flowchart are shown in Figure 1. A total number of 1958 studies, 946 in PubMed and 1012 in EMBASE, were identified in the databases. Af- ter removing the duplicates, 1776 articles were screened, checking titles and abstracts according to the selection criteria (Table II). Eighty articles were screened as fulltext assessment, of which 31 studies were selected for this systematic literature review.

Systemic lupus erythematosus was the most investigated disease in terms of treatment adherence with 26 studies $(83 \%)$. Three studies $(9 \%)$ were conducted in Behçet's disease patients, while two studies were focused on systemic sclerosis. A summary of the results of the selected studies is presented in Table III (18-48).

Table III - Summary of the selected studies. Studies are ordered by publication year and the alphabetic authors name order.

\begin{tabular}{|c|c|c|c|c|c|c|c|}
\hline Authors & Title & Journal & Year & Disease & $\begin{array}{l}\text { No. of } \\
\text { patients }\end{array}$ & Tools/method & $\begin{array}{c}\text { Adherence } \\
\text { rate }(\%)\end{array}$ \\
\hline $\begin{array}{l}\text { DuX, Chen H, Zhuang Y, } \\
\text { Zhao Q, Shen B. (18) }\end{array}$ & $\begin{array}{l}\text { Medication Adherence in Chinese Patients with } \\
\text { Systemic Lupus Erythematosus }\end{array}$ & $\begin{array}{l}J \text { Clin } \\
\text { Rheumatol }\end{array}$ & 2020 & $\begin{array}{l}\text { Systemic lupus } \\
\text { erythematosus } \\
\text { (SLE) }\end{array}$ & 144 & $\begin{array}{l}\text { Compliance } \\
\text { questionnaire } \\
\text { rheumatology } \\
\text { (cqr) }\end{array}$ & $43 \%$ \\
\hline $\begin{array}{l}\text { E Hachulla, N LeGouellec, } \\
\text { D Launay, Marie-Hélène } \\
\text { Balquet, et al. (19) }\end{array}$ & $\begin{array}{l}\text { Adherence to hydroxychloroquine in patients with } \\
\text { systemic lupus: contrasting results and weak } \\
\text { correlation between assessment tools }\end{array}$ & $\begin{array}{l}\text { Joint Bone } \\
\text { Spine }\end{array}$ & 2020 & $\begin{array}{l}\text { Systemic lupus } \\
\text { erythematosus } \\
\text { (SLE) }\end{array}$ & 145 & $\begin{array}{l}\text { Medication } \\
\text { Adherence } \\
\text { Self-Report } \\
\text { Inventory (MASRI) } \\
\text { and Morisky } \\
\text { Medication } \\
\text { Adherence Scales } \\
\text { (MMAS-8) }\end{array}$ & $63 \%$ \\
\hline $\begin{array}{l}\text { Harry 0, Crosby LE, Mara } \\
\text { C, Ting T V, Huggins JL, } \\
\text { Modi AC. (20) }\end{array}$ & $\begin{array}{l}\text { Feasibility and acceptability of an innovative } \\
\text { adherence intervention for young adults with } \\
\text { childhood-onset systemic Lupus Erythematosus }\end{array}$ & $\begin{array}{l}\text { Pediatr } \\
\text { Rheumatol }\end{array}$ & 2020 & $\begin{array}{l}\text { Systemic lupus } \\
\text { erythematosus } \\
\text { (SLE) }\end{array}$ & 19 & $\begin{array}{l}\text { Medication } \\
\text { Adherence Self- } \\
\text { Report Inventory } \\
\text { (MASRI) }\end{array}$ & NA \\
\hline $\begin{array}{l}\text { Ali AY, Abdelaziz TS, } \\
\text { Essameldin M. (21) }\end{array}$ & $\begin{array}{l}\text { The prevalence and causes non-adherence to } \\
\text { immunosuppressive medications in patients with } \\
\text { lupus nephritis flares }\end{array}$ & $\begin{array}{l}\text { Curr Rheumatol } \\
\text { Rev }\end{array}$ & 2019 & $\begin{array}{l}\text { Systemic lupus } \\
\text { erythematosus } \\
\text { (SLE) }\end{array}$ & 104 & $\begin{array}{l}\text { Morisky } \\
\text { Medication } \\
\text { Adherence Scales } \\
\text { (MMAS-8) }\end{array}$ & $35 \%$ \\
\hline $\begin{array}{l}\text { Brijs J, Arat S, Westhovens } \\
\text { R, Lenaerts JL, De Langhe } \\
\text { E. (22) }\end{array}$ & $\begin{array}{l}\text { Treatment adherence in systemic sclerosis: A } \\
\text { cross-sectional study }\end{array}$ & $\begin{array}{l}\text { Musculoskeletal } \\
\text { Care }\end{array}$ & 2019 & $\begin{array}{l}\text { Systemic } \\
\text { sclerosis }\end{array}$ & 66 & $\begin{array}{l}\text { Compliance } \\
\text { questionnaire } \\
\text { rheumatology } \\
\text { (cqr) }\end{array}$ & $28 \%$ \\
\hline $\begin{array}{l}\text { Clowse MEB, Eudy AM, } \\
\text { Revels J, Neil L, Sanders } \\
\text { GD. (23) }\end{array}$ & $\begin{array}{l}\text { Provider perceptions on the management of } \\
\text { lupus during pregnancy: barriers to improved care }\end{array}$ & Lupus & 2019 & $\begin{array}{l}\text { Lupus during } \\
\text { pregnancy }\end{array}$ & 0 & $\begin{array}{l}\text { PRECEDE/ } \\
\text { PROCEED } \\
\text { framework }\end{array}$ & NA \\
\hline $\begin{array}{l}\text { Amalia R, Sasongko H, } \\
\text { Kundarto W, Niruri R. (24) }\end{array}$ & $\begin{array}{l}\text { The analysis of the factors affecting medication } \\
\text { adherence in patient with SLE (Systemic Lupus } \\
\text { Erythematosus) at Yayasan Tittari Griya Kupu } \\
\text { Solo }\end{array}$ & $\begin{array}{l}\text { Indian J Public } \\
\text { Heal Res Dev }\end{array}$ & 2019 & $\begin{array}{l}\text { Systemic lupus } \\
\text { erythematosus } \\
\text { (SLE) }\end{array}$ & 41 & $\begin{array}{l}\text { Morisky green } \\
\text { levine scale (mgls) }\end{array}$ & $36 \%$ \\
\hline $\begin{array}{l}\text { Sun K, Eudy AM, Rogers } \\
\text { JL, Criscione-Schreiber } \\
\text { LG, Doss J, Sadun RE, } \\
\text { et al. (25) }\end{array}$ & $\begin{array}{l}197 \text { Racial disparities in lupus medication } \\
\text { adherence }\end{array}$ & Lupus Sci Med & 2019 & $\begin{array}{l}\text { Systemic lupus } \\
\text { erythematosus } \\
\text { (SLE) }\end{array}$ & 84 & $\begin{array}{l}\text { Medication } \\
\text { Adherence Self- } \\
\text { Report Inventory } \\
\text { (MASRI) }\end{array}$ & $50 \%-64 \%$ \\
\hline
\end{tabular}




\begin{tabular}{|c|c|c|c|c|c|c|c|}
\hline Authors & Title & Journal & Year & Disease & $\begin{array}{l}\text { No. of } \\
\text { patients }\end{array}$ & Tools/method & $\begin{array}{c}\text { Adherence } \\
\text { rate }(\%)\end{array}$ \\
\hline $\begin{array}{l}\text { Wallace DJ, Tse K, } \\
\text { Hanrahan L, Davies R, } \\
\text { Petri MA. (26) }\end{array}$ & $\begin{array}{l}\text { Hydroxychloroquine usage in US patients, } \\
\text { their experiences of tolerability and adherence, } \\
\text { and implications for treatment: Survey results } \\
\text { from } 3127 \text { patients with SLE conducted by the } \\
\text { Lupus Foundation of America }\end{array}$ & Lupus Sci Med & 2019 & $\begin{array}{l}\text { Systemic lupus } \\
\text { erythematosus } \\
\text { (SLE) }\end{array}$ & 2783 & $\begin{array}{l}\mathrm{HCQ} \text { dosage } \\
\text { questionnaire }\end{array}$ & NA \\
\hline $\begin{array}{l}\text { Zayed HS, Medhat BM, } \\
\text { Seif EM. (27) }\end{array}$ & $\begin{array}{l}\text { Evaluation of treatment adherence in patients } \\
\text { with Behçet's disease: its relation to disease } \\
\text { manifestations, patients' beliefs about } \\
\text { medications, and quality of life }\end{array}$ & Clin Rheumatol & 2019 & $\begin{array}{l}\text { Behçet's } \\
\text { disease (BD) }\end{array}$ & 67 & $\begin{array}{l}\text { Compliance } \\
\text { questionnaire of } \\
\text { rheumatology } \\
\text { (CQR) }\end{array}$ & $22 \%$ \\
\hline $\begin{array}{l}\text { Khabbazi A, Karkon } \\
\text { Shayan F, Ghojazadeh M, } \\
\text { Kavandi H, Hajialiloo M, } \\
\text { Esalat Manesh K, et al. (28) }\end{array}$ & $\begin{array}{l}\text { Adherence to treatment in patients with } \\
\text { Behçet's disease }\end{array}$ & Int J Rheum Dis & 2018 & $\begin{array}{l}\text { Behçet's } \\
\text { disease (BD) }\end{array}$ & 137 & $\begin{array}{l}\text { Self-reported } \\
\text { questionnaire }\end{array}$ & $50 \%$ \\
\hline $\begin{array}{l}\text { Georgopoulou S, Nel L, } \\
\text { Sangle S, Robson M, } \\
\text { D'Cruz DP. (29) }\end{array}$ & $\begin{array}{l}\text { Physician-patient interaction and medication } \\
\text { adherence in lupus nephritis }\end{array}$ & Rheumatology & 2018 & $\begin{array}{l}\text { Systemic lupus } \\
\text { erythematosus } \\
\text { (SLE) }\end{array}$ & 98 & $\begin{array}{l}\text { Self-reported } \\
\text { questionnaire }\end{array}$ & NA \\
\hline $\begin{array}{l}\text { Costedoat-Chalumeau N, } \\
\text { Houssiau F, Izmirly P, Le } \\
\text { Guern V, Navarra S, Jolly } \\
\text { M, et al. (30) }\end{array}$ & $\begin{array}{l}\text { A Prospective International Study on } \\
\text { Adherence to Treatment in } 305 \text { Patients with } \\
\text { Flaring SLE: Assessment by Drug Levels and } \\
\text { Self-Administered Questionnaires }\end{array}$ & $\begin{array}{l}\text { Clin Pharmacol } \\
\text { Ther }\end{array}$ & 2018 & $\begin{array}{l}\text { Systemic lupus } \\
\text { erythematosus } \\
\text { (SLE) }\end{array}$ & 305 & $\begin{array}{l}\text { Medication } \\
\text { Adherence } \\
\text { Self-Report } \\
\text { Inventory (MASRI) } \\
\text { \& Morisky } \\
\text { Medication } \\
\text { Adherence Scales } \\
\text { (MMAS-8) }\end{array}$ & $61 \%$ \\
\hline $\begin{array}{l}\text { ludici M, Pantano I, } \\
\text { Fasano S, Pierro L, } \\
\text { Charlier B, Pingeon M, et } \\
\text { al. (31) }\end{array}$ & $\begin{array}{l}\text { Health status and concomitant prescription } \\
\text { of immunosuppressants are risk factors } \\
\text { for hydroxychloroquine non-adherence in } \\
\text { systemic lupus patients with prolonged } \\
\text { inactive disease }\end{array}$ & Lupus & 2018 & $\begin{array}{l}\text { Systemic lupus } \\
\text { erythematosus } \\
\text { (SLE) }\end{array}$ & 83 & $\begin{array}{l}\text { HCQ dosage } \\
\text { questionnaire and } \\
\text { blood samples }\end{array}$ & $71 \%$ \\
\hline $\begin{array}{l}\text { Alsowaida N, Alrasheed } \\
\text { M, Mayet A, Alsuwaida A, } \\
\text { Omair MA. (32) }\end{array}$ & $\begin{array}{l}\text { Medication adherence, depression and } \\
\text { disease activity among patients with systemic } \\
\text { lupus erythematosus }\end{array}$ & Lupus & 2018 & $\begin{array}{l}\text { Systemic lupus } \\
\text { erythematosus } \\
\text { (SLE) }\end{array}$ & 140 & $\begin{array}{l}\text { Morisky } \\
\text { medication } \\
\text { adherence scales } \\
\text { (mmas-4) }\end{array}$ & $38 \%$ \\
\hline $\begin{array}{l}\text { Kenneth Johnsen, } \\
\text { Meenakshi Jolly, Narender } \\
\text { Annapureddy. (33) }\end{array}$ & $\begin{array}{l}\text { Health related quality of life in lupus: Self- } \\
\text { management-a modifiable predictor }\end{array}$ & $\begin{array}{l}\text { ACR/ARHP } \\
\text { Annual Meeting }\end{array}$ & 2017 & $\begin{array}{l}\text { Systemic lupus } \\
\text { erythematosus } \\
\text { (SLE) }\end{array}$ & 50 & $\begin{array}{l}\text { Medication } \\
\text { Adherence Self- } \\
\text { Report Inventory } \\
\text { (MASRI) }\end{array}$ & NA \\
\hline $\begin{array}{l}\text { Alexandra Perel-Winkler, } \\
\text { Kayla Neville, Samantha } \\
\text { Nguyen, Miya Okado, } \\
\text { James Miceli, Jon T. Giles, } \\
\text { Anca Askanase. (34) }\end{array}$ & $\begin{array}{l}\text { Low health literacy does not impact } \\
\text { adherence to hydroxychloroquine in patients } \\
\text { with systemic lupus }\end{array}$ & $\begin{array}{l}\text { ACR/ARHP } \\
\text { Annual Meeting }\end{array}$ & 2017 & $\begin{array}{l}\text { Systemic lupus } \\
\text { erythematosus } \\
\text { (SLE) }\end{array}$ & 67 & $\begin{array}{l}\text { Self-reported } \\
\text { questionnaire }\end{array}$ & NA \\
\hline $\begin{array}{l}\text { Farinha F, Freitas F, } \\
\text { Águeda A, Cunha I, } \\
\text { Barcelos A. (35) }\end{array}$ & $\begin{array}{l}\text { Concerns of patients with systemic lupus } \\
\text { erythematosus and adherence to therapy - a } \\
\text { qualitative study }\end{array}$ & $\begin{array}{l}\text { Patient Prefer } \\
\text { Adherence }\end{array}$ & 2017 & $\begin{array}{l}\text { Systemic lupus } \\
\text { erythematosus } \\
\text { (SLE) }\end{array}$ & 15 & $\begin{array}{l}\text { Semi-structured } \\
\text { face-to-face } \\
\text { interviews }\end{array}$ & NA \\
\hline $\begin{array}{l}\text { Cinar M, Cinar Fl, Acikel C, } \\
\text { Yilmaz S, Cakar M, Horne } \\
\text { R, et al. (36) }\end{array}$ & $\begin{array}{l}\text { Reliability and validity of the Turkish translation } \\
\text { of the beliefs about medicines questionnaire } \\
\text { (BMQ-T) in patients with Behçet's disease }\end{array}$ & $\begin{array}{l}\text { Clin Exp } \\
\text { Rheumatol }\end{array}$ & 2016 & $\begin{array}{l}\text { Behçet's } \\
\text { disease (BD) }\end{array}$ & 125 & $\begin{array}{l}\text { Beliefs about } \\
\text { Medicines } \\
\text { Questionnaire } \\
\text { (BMQ) }\end{array}$ & NA \\
\hline $\begin{array}{l}\text { Flower C, Hambleton I, } \\
\text { Campbell M. (37) }\end{array}$ & $\begin{array}{l}\text { The Effect of Psychosocial and } \\
\text { Neuropsychiatric Factors on Medication } \\
\text { Adherence in a Cohort of Women with } \\
\text { Systemic Lupus Erythematosus }\end{array}$ & $\begin{array}{l}\text { J Clin } \\
\text { Rheumatol }\end{array}$ & 2016 & $\begin{array}{l}\text { Systemic lupus } \\
\text { erythematosus } \\
\text { (SLE) }\end{array}$ & 106 & $\begin{array}{l}\text { Morisky } \\
\text { medication } \\
\text { adherence scales } \\
\text { (mmas-4) }\end{array}$ & $60 \%$ \\
\hline
\end{tabular}




\begin{tabular}{|c|c|c|c|c|c|c|c|}
\hline Authors & Title & Journal & Year & Disease & $\begin{array}{l}\text { No. of } \\
\text { patients }\end{array}$ & Tools/method & $\begin{array}{l}\text { Adherence } \\
\text { rate }(\%)\end{array}$ \\
\hline $\begin{array}{l}\text { Cristina Drenkard, Sonia } \\
\text { Mathew, Gaobin Bao. S. } \\
\text { Sam Lim. (38) }\end{array}$ & $\begin{array}{l}\text { Depression Is a Risk Factor for Low Treatment } \\
\text { Adherence in African American People with } \\
\text { Systemic Lupus Erythematosus }\end{array}$ & $\begin{array}{l}\text { ACR/ARHP } \\
\text { Annual Meeting }\end{array}$ & 2016 & $\begin{array}{l}\text { Systemic lupus } \\
\text { erythematosus } \\
\text { (SLE) }\end{array}$ & 326 & $\begin{array}{l}\text { Morisky } \\
\text { Medication } \\
\text { Adherence Scales } \\
\text { (MMAS-8) }\end{array}$ & $46 \%$ \\
\hline $\begin{array}{l}\text { Singh JA, Qu H, Yazdany } \\
\text { J, Chatham W, Dall'Era M, } \\
\text { Shewchuk RM. (39) }\end{array}$ & $\begin{array}{l}\text { Barriers to medication decision making in women } \\
\text { with lupus nephritis: A formative study using } \\
\text { nominal group technique }\end{array}$ & J Rheumatol & 2015 & $\begin{array}{l}\text { Systemic lupus } \\
\text { erythematosus } \\
\text { (SLE) }\end{array}$ & 51 & $\begin{array}{l}\text { Nominal group } \\
\text { technique (NGT) }\end{array}$ & NA \\
\hline $\begin{array}{l}\text { Hale ED, Radvanski DC, } \\
\text { Hassett AL. (40) }\end{array}$ & $\begin{array}{l}\text { The man-in-the-moon face: a qualitative study } \\
\text { of body image, self-image and medication use in } \\
\text { systemic lupus erythematosus }\end{array}$ & Rheumatology & 2015 & $\begin{array}{l}\text { Systemic lupus } \\
\text { erythematosus } \\
\text { (SLE) }\end{array}$ & 15 & $\begin{array}{l}\text { Semi-structured } \\
\text { face-to-face } \\
\text { interviews }\end{array}$ & NA \\
\hline $\begin{array}{l}\text { Hromadkova L, Soukup T, } \\
\text { Cermakova E, Vlcek J. (41) }\end{array}$ & $\begin{array}{l}\text { Drug compliance in patients with systemic } \\
\text { scleroderma }\end{array}$ & Clin Rheumatol & 2012 & $\begin{array}{l}\text { Systemic } \\
\text { sclerosis }\end{array}$ & 41 & $\begin{array}{l}\text { Compliance } \\
\text { questionnaire } \\
\text { rheumatology } \\
\text { (cqr) }\end{array}$ & $75 \%$ \\
\hline $\begin{array}{l}\text { Daleboudt GMN, } \\
\text { Broadbent E, McQueen F, } \\
\text { Kaptein AA, et al. (42) }\end{array}$ & $\begin{array}{l}\text { Intentional and unintentional treatment } \\
\text { nonadherence in patients with systemic lupus } \\
\text { erythematosus }\end{array}$ & $\begin{array}{l}\text { Arthritis Care } \\
\text { Res }\end{array}$ & 2011 & $\begin{array}{l}\text { Systemic lupus } \\
\text { erythematosus } \\
\text { (SLE) }\end{array}$ & 106 & $\begin{array}{l}\text { Self-reported } \\
\text { questionnaire }\end{array}$ & $86 \%$ \\
\hline $\begin{array}{l}\text { Duvdevany I, Cohen M, } \\
\text { Minsker-Valtzer A, Lorber } \\
\text { M. (43) }\end{array}$ & $\begin{array}{l}\text { Psychological correlates of adherence to self- } \\
\text { care, disease activity and functioning in persons } \\
\text { with systemic lupus erythematosus }\end{array}$ & Lupus & 2011 & $\begin{array}{l}\text { Systemic lupus } \\
\text { erythematosus } \\
\text { (SLE) }\end{array}$ & 100 & $\begin{array}{l}\text { Self-reported } \\
\text { questionnaire }\end{array}$ & NA \\
\hline $\begin{array}{l}\text { Bennett JK, Fuertes JN, } \\
\text { Keitel M, Phillips R, et } \\
\text { al. (44) }\end{array}$ & $\begin{array}{l}\text { The role of patient attachment and working } \\
\text { alliance on patient adherence, satisfaction, and } \\
\text { health-related quality of life in lupus treatment }\end{array}$ & $\begin{array}{l}\text { Patient Educ } \\
\text { Couns }\end{array}$ & 2011 & $\begin{array}{l}\text { Systemic lupus } \\
\text { erythematosus } \\
\text { (SLE) }\end{array}$ & 193 & $\begin{array}{l}\text { General } \\
\text { adherence } \\
\text { inventory }\end{array}$ & NA \\
\hline $\begin{array}{l}\text { Chambers SA, Raine } \\
\text { R, Rahman A, Isenberg } \\
\text { D. (45) }\end{array}$ & $\begin{array}{l}\text { Why do patients with systemic lupus } \\
\text { erythematosus take or fail to take their prescribed } \\
\text { medications? A qualitative study in a UK cohort }\end{array}$ & Rheumatology & 2009 & $\begin{array}{l}\text { Systemic lupus } \\
\text { erythematosus } \\
\text { (SLE) }\end{array}$ & 315 & $\begin{array}{l}\text { Self-reported } \\
\text { questionnaire }\end{array}$ & NA \\
\hline $\begin{array}{l}\text { Chambers S, Raine R, } \\
\text { Rahman A, Hagley K, De } \\
\text { Ceulaer K, Isenberg D. (46) }\end{array}$ & $\begin{array}{l}\text { Factors influencing adherence to medications } \\
\text { in a group of patients with systemic lupus } \\
\text { erythematosus in Jamaica }\end{array}$ & Lupus & 2008 & $\begin{array}{l}\text { Systemic lupus } \\
\text { erythematosus } \\
\text { (SLE) }\end{array}$ & 75 & $\begin{array}{l}\text { Self-reported } \\
\text { questionnaire }\end{array}$ & $56 \%$ \\
\hline $\begin{array}{l}\text { Koneru S, Kocharla L, } \\
\text { Higgins GC, Ware A, } \\
\text { Passo MH, Farhey YD, et } \\
\text { al. (47) }\end{array}$ & $\begin{array}{l}\text { Adherence to medications in systemic lupus } \\
\text { erythematosus }\end{array}$ & $\begin{array}{l}\text { J Clin } \\
\text { Rheumatol }\end{array}$ & 2008 & $\begin{array}{l}\text { Systemic lupus } \\
\text { erythematosus } \\
\text { (SLE) }\end{array}$ & 63 & $\begin{array}{l}\text { Medication } \\
\text { Adherence Self- } \\
\text { Report Inventory } \\
\text { (MASRI) }\end{array}$ & $61 \%$ \\
\hline $\begin{array}{l}\text { Garcia Popa-Lisseanu, } \\
\text { Greisinger M, Richardson } \\
\text { A, et al. (48) }\end{array}$ & $\begin{array}{l}\text { Determinants of treatment adherence in ethnically } \\
\text { diverse, economically disadvantaged patients } \\
\text { with rheumatic disease }\end{array}$ & J Rheumatol & 2005 & $\begin{array}{l}\text { Systemic lupus } \\
\text { erythematosus } \\
\text { (SLE) }\end{array}$ & 22 & Focus groups & NA \\
\hline
\end{tabular}

Self-reported measurements, such as in interviews, self-reported questionnaires and focus groups in which patients provided information about medication they were taking, emerged as the most practical and common approaches to measure the adherence rate in CTDs $(28,29,34,35,39,40$, $42,43,45,46,48)$. In particular, seven studies developed a tailored self-reported questionnaire to assess medication adherence. For instance, Chambers et al. (46) developed their own qualitative study using interviews and questionnaires covering so- cio-demographic information such as: age, sex, ethnic group, employment status, religion and treatment details. The questionnaire also allowed the authors to estimate, by asking respondents, the percentage of days related to the previous six months in which patients took all their prescribed medicines. These tools revealed that the main reasons for poor medication adherence were the cost and the setting-related availability of the medication. Patient interviews are generally considered practical low-cost tools that, under certain condi- 
tions, can explore the reasons behind behaviors of patient in taking medication, thus providing explanations for any difficulties in following their treatment regimens. Even if the questionnaires address specific aspects related to condition fac- tors, which cannot be explored with other types of general medication adherence questionnaires, none of the seven questionnaires can be considered a gold standard approach to assess medication adherence. Moreover, none of them was developed in

Table IV - Summary table of questionnaire domain exploration.

\begin{tabular}{|c|c|c|c|c|}
\hline $\begin{array}{l}\text { Questionnaire/ } \\
\text { tool }\end{array}$ & $\begin{array}{l}\text { Number of } \\
\text { questions }\end{array}$ & Domain & Advantages & Limitations \\
\hline $\begin{array}{l}\text { Brief } \\
\text { Medication } \\
\text { Questionnaire } \\
\text { (BMQ) }\end{array}$ & 9 questions & $\begin{array}{l}\text { The Brief Medication Questionnaire (BMQ) is composed of } \\
9 \text {-item, can explore both patients taking behavior and barriers } \\
\text { to adherence. Includes } 5 \text {-item for regimen screening, 2-item for } \\
\text { belief screen about drug effects, and 2-item recall difficulties } \\
\text { in remembering. One of the main advantages is that it can } \\
\text { evaluate multi-drug regimens. However, it is time-consuming } \\
\text { compared with other questionnaires, therefore it is difficult to } \\
\text { use at the point of care. }\end{array}$ & $\begin{array}{l}\text { It evaluates multi- } \\
\text { drug regimens. } \\
\text { It explores patients } \\
\text { taking behavior } \\
\text { and barriers to } \\
\text { adherence. }\end{array}$ & $\begin{array}{l}\text { Time-consuming } \\
\text { Complicated } \\
\text { scoring system }\end{array}$ \\
\hline $\begin{array}{l}\text { Compliance } \\
\text { questionnaire } \\
\text { rheumatology } \\
\text { (CQR) }\end{array}$ & 19 questions & $\begin{array}{l}\text { The CQR is a patient-oriented questionnaire rheumatology- } \\
\text { specific tool that measures patient adherence to drug } \\
\text { regimens, detects factors that contribute to sub-optimal } \\
\text { patient compliance. When used in conjunction with specialized } \\
\text { psychosocial measures, it provides information such as the } \\
\text { barriers to taking medication. }\end{array}$ & $\begin{array}{l}\text { It encourages } \\
\text { psychometric } \\
\text { properties. } \\
\text { Indication of the } \\
\text { social or cognitive } \\
\text { reasons behind } \\
\text { non-adherence. }\end{array}$ & $\begin{array}{l}\text { Time-consuming. } \\
\text { Long series of } \\
\text { questions. } \\
\text { The discriminant } \\
\text { ability does not } \\
\text { correlate well in } \\
\text { either compliance } \\
\text { or correct dosing. }\end{array}$ \\
\hline $\begin{array}{l}\text { Medication } \\
\text { Adherence } \\
\text { Self-Report } \\
\text { Inventory } \\
\text { (MASRI) }\end{array}$ & 12 questions & $\begin{array}{l}\text { The Medication Adherence Self-Report Inventory (MASRI) is } \\
\text { a self-administered questionnaire, easily manageable in the } \\
\text { clinical setting. It offers an accurate measurement of adherence } \\
\text { to the drug therapy in systemic lupus erythematosus (SLE). } \\
\text { MASRI addresses two broad themes: the amount of medication } \\
\text { taken and the timing of the doses. However, MASRI does not } \\
\text { allow the assessment of medication overuse. }\end{array}$ & $\begin{array}{l}\text { Easy and } \\
\text { manageable in } \\
\text { clinical setting. } \\
\text { Suitable for } \\
\text { systemic lupus } \\
\text { erythematosus. }\end{array}$ & $\begin{array}{l}\text { Time-consuming. } \\
\text { Not consider } \\
\text { medication overuse. }\end{array}$ \\
\hline $\begin{array}{l}\text { Morisky } \\
\text { Medication } \\
\text { Adherence } \\
\text { Scale } \\
\text { (MMAS-8) }\end{array}$ & 8 que & $\begin{array}{l}\text { The Morisky Medication Adherence Scale (MMAS-8) developed } \\
\text { from the original four-item MGLS is the most used questionnaire } \\
\text { for medication adherence assessment. It identifies reasons for } \\
\text { medication non-adherence and has better internal consistency. } \\
\text { Nevertheless, like the MGLS, MMAS- } 8 \text { has disadvantages, such } \\
\text { as capturing only a few factors associated with non-adherence, } \\
\text { thus providing limited information to develop interventions } \\
\text { targeting non-adherence. }\end{array}$ & $\begin{array}{l}\text { It captures the } \\
\text { behaviour of } \\
\text { patients in taking } \\
\text { medications. } \\
\text { High internal } \\
\text { consistency. }\end{array}$ & $\begin{array}{l}\text { Expensive } \\
\text { Limited ability } \\
\text { to capture non- } \\
\text { adherence reasons. }\end{array}$ \\
\hline $\begin{array}{l}\text { Morisky } \\
\text { Medication } \\
\text { Adherence } \\
\text { Scale } \\
\text { (MMAS-4) }\end{array}$ & 4 questions & $\begin{array}{l}\text { Four dichotomous items compose the 4-item Morisky } \\
\text { Medication Adherence Scale (MMAS-4), offering an easy and } \\
\text { practical application in the clinical setting. Like in the MMAS-8, } \\
\text { it is able to capture the medication taking behaviour of patients, } \\
\text { but not properly assess the reasons or predictors of medication } \\
\text { adherence. }\end{array}$ & $\begin{array}{l}\text { High validity and } \\
\text { reliability. } \\
\text { Short } \\
\text { questionnaire. }\end{array}$ & $\begin{array}{l}\text { Expensive. } \\
\text { Limited ability } \\
\text { to capture non- } \\
\text { adherence reasons. }\end{array}$ \\
\hline $\begin{array}{l}\text { Morisky green } \\
\text { levine scale } \\
\text { (mgls) }\end{array}$ & 4 questions & $\begin{array}{l}\text { Designed as a four-item scale, the Morisky Green and Levine } \\
\text { (MGLS) Medication Adherence Scale is a dichotomous } \\
\text { questionnaire based on "yes" or "no" response categories. } \\
\text { MGLS can address barriers to medication-taking and permit the } \\
\text { health care provider to reinforce positive adherence behaviors. } \\
\text { However, in some studies MGLS has shown poor consistency } \\
\text { when used in an elderly population. In particular, as a single } \\
\text { measure of adherence it does not show an acceptable level of } \\
\text { reliability. }\end{array}$ & $\begin{array}{l}\text { Short } \\
\text { questionnaire. } \\
\text { Easily integrated } \\
\text { into the medical } \\
\text { visit }\end{array}$ & $\begin{array}{l}\text { It fails to identify } \\
\text { partial adherence. } \\
\text { Patient's desirability } \\
\text { bias } \\
\text { It lacks consistency }\end{array}$ \\
\hline
\end{tabular}


co-design with patients or with any other stakeholder. In addition, the drawback of this approach is the relatively poor sensitivity and consistency of the questionnaire leading to possible skewed results $(49,50)$. The second set of tools was identified, relying on structured and validated questionnaires. The Medication Adherence SelfReport Inventory (MASRI) (51), the 4-item (52) and 8-item (53) Morisky Medication Adherence Scale (MMAS), and the Brief Medication Questionnaire (BMQ) (54), were identified as the most used and structured set of tools found in our results. These tools apply cut-off values or rank the degree of the medication adherence rate. Such approaches can minimize the limitations of other self-reported methods by standardizing the measurement and increasing the validation consistency. These questionnaires are often validated with statistical tests and combined with other subjective or objective measures, thus improving the consistency of the results (55). The MASRI questionnaire was adopted in five papers $(20,25,30,33,47)$ and all studies explored the adherence rate in SLE. The CQR was used in four publications $(18,22$, 27,41 not only in lupus, but also in systemic sclerosis and Behçet's disease, given it is specific for rheumatological disorders. The eight items of the MMAS were used in three papers $(21,30,38)$, while the version with 4 items was used in two more papers $(32,37)$. It is important to highlight that the 8-item MMAS was particularly valuable in these studies, as it makes it possible to recognize whether the causes of non-adherence are intentional or non-intentional, demonstrating the added value of this tool also regarding patient beliefs about medicine. A tool for assessing patient beliefs about medicine is the BMQ, used by Çinar et al. (36) who validated its Turkish translation.

Alternative tools with other tailored characteristics, such as exploring specific factors related to the disease(s) or treatments, were adopted in three studies $(26,31,44)$. These tools can be considered valuable alternatives to investigate specific variables that may not be captured with standard tools, even if further validation might be useful to enhance consistency and ensure their implementation.

An interesting research was recently published regarding adherence assessment of SLE patients in France (19), which showed how the combination of different tools with direct and indirect measurements can provide an overall estimation of the magnitude and reasons behind non-adherence. Specifically, the authors reported that the combination of blood hydroxychloroquine concentration with MASRI and MMAS-8 may help to better identify non-adherence in SLE.

The ideal medication adherence assessment tool should have a user-friendly design, be easy and practical to use in any clinical settings and offer high reliability and consistency. However, this is not yet reality, since so far no tool can meet all these standards at the same time and each tool has its own advantages and drawbacks.

In summary, Table IV reports the advantages, limitations, and specific domain of each of the main questionnaires showing at a glance all the differences across the various medication adherence tools identified.

\section{DISCUSSION}

Medication adherence is a pivotal problem in the management of connective tissue diseases. The results of this study demonstrated the existence of valid tools aimed at measuring the medication adherence rate. Since the ideal measurement tool should be as much flexible, reliable, and practical as possible for the clinical setting as well as for the patient, the existing tools can be considered appropriate to measure the adherence rate in CTDs. However, only a few CTDs have been extensively studied so far, in particular, SLE, Behçet's disease and systemic sclerosis.

Across all the included studies, a wide range of tools and methods were found to assess medication adherence. Each tool has pros and cons and explores important dimensions to mitigate patient non-adherence barriers. Although the included studies met standards for describing rationale 
and methods, existing studies are not yet involving patients and their representatives in the co-design phase during the methodological development. Such an approach, in our opinion, can provide a significant added value in identifying the boundaries of non-adherence at the patient level, and the associated modifiable factors.

The MMAS in both versions, (MMAS-4) and (MMAS-8), is a well-known tool used to measure adherence in patients with chronic conditions. The original version with four items developed by Morisky was quite innovative in capturing the fundamental reasons for non-adherence (56). The eight items (MMAS-8) version developed in 2008 improved the psychometric properties, increased sensitivity and specificity, as reported in several studies, and was translated into many languages (57). MMAS-8 is the most widely used self-reported measurement for medication adherence assessment. Another relevant tool is the MASRI, a concise self-administered tool composed of 12 items which can be easily administered in the clinical setting and is effective in SLE drug therapy assessment (58). Originally used in HIV therapy, the MASRI questionnaire has proven useful for adherence assessment in SLE, adopting a visual analogue scale (VAS) item which is known and massively used in rheumatologic settings to estimate a numeric value of the adherence rate $(0-100 \%)$.

In addition, the CQR (59) is a validated tool for measuring adherence in rheumatic diseases. CQR can identify factors that contribute to sub-optimal adherence like social or cognitive factors. It was validated against the medication event monitoring system (eMEMs), which is an electronic cap fitted on standard drug bottles indicating the time and date on which the bottle was opened. The CQR questions were identified through focus groups and clinical expert opinions. Despite this tool has proven valuable, it can be difficult to apply routinely in some clinical settings, as it consists of 19 items (60).

Only a few studies explored the efficacy of educational interventions aimed at improving the adherence to treatment. These stud- ies yielded some heterogeneous results that could encourage the implementation of projects which involve patients in the codesign of these interventions. As a matter of fact, evidence clearly suggests that the lack of patient engagement and confidence plays an important role (61). On the other side, motivation and support in meeting their needs with tools resulting from a codesign process (62) could increase patient confidence and knowledge in addressing such complex issues.

Many of the studies reported in this paper have used a combination of tools, including blood samples as a direct approach to explore medication adherence.

The provided evidence suggests clearly that a low medication adherence rate is a reality also among patients with CTDs, similarly to other chronic diseases. For this reason, one of the priorities that should be considered is the co-creation and organization of initiatives aiming to improve the relevance of adherence measurement and to promote patient education. For this purpose, existing tools could be reinforced with additional features aimed at exploring in greater detail the real reasons and barriers behind low- or non-adherence. The identification of these issues should be considered the basis for planning and implementing tailored actions and initiatives specifically aimed at improving medication adherence in CTDs. Since CTDs are heterogeneous and can include different phenotypes, the reasons behind low- and nonadherence can be really variable not only in the different diseases, but also in the different subsets of the same disease (e.g. low- or non-adherence related to disease remission is very different from low- or non-adherence due to being scared by possible adverse drug reactions). The development of additional tailored tools created in co-design with all the stakeholders involved in adherence management would be crucial to ensure that the various dimensions and barriers leading to low- and non-adherence can be identified and addressed in actions aimed at improving adherence in CTDs patients. This kind of approach is even more important in CTDs because the complexity 
of these diseases and the low number of patients cannot guarantee enough information for strong statistical assumptions (63).

\section{CONCLUSIONS}

Low- and non-adherence were explored in some CTDs with valid and useful tools, while other CTDs still need to be assessed. Therefore, more efforts should be focused on better understanding the specific reasons for the low- and adherence in CTDs patients. Moreover, additional tools should be developed in co-design with patients and other stakeholders in order to provide a comprehensive approach (64) to include the different types of dimensions, such as: beliefs, personal preference, social support, disease conditions and patient-doctor and patient-caregiver relationships. The limitations and barriers related to low- and non- adherence can be then addressed in specific co-designed initiatives that can increase adherence and, therefore, improve not only health outcomes, but also the quality of life of patients affected by CTDs.

\section{Acknowledgments}

Special thanks go to the entire ERN ReCONNET research team for the commitment to support this work.

\section{Disclosure}

The authors declare no conflicts of interest.

\section{REFERENCES}

1. Sabaté E, World Health Organization. Adherence to long-term therapies: evidence for action. World Health Organization; 2003, pp. 194. Available from: https://apps.who.int/ medicinedocs/en/d/Js4883e/

2. Coulter A, Entwistle VA, Eccles A, et al. Personalised care planning for adults with chronic or long-term health conditions. Cochrane Database Syst Rev. 2015; (3): CD010523.

3. Haynes RB, Ackloo E, Sahota N, McDonald HP, Yao X. Interventions for enhancing medication adherence. Cochrane Database Syst Rev. 2008; (2): CD000011.

4. Golay A. Pharmacoeconomic aspects of poor adherence: can better adherence reduce healthcare costs? J Med Econ. 2011; 14: 594-608.

5. Lee JK, Grace KA, Taylor AJ. Effect of a pharmacy care program on medication adher- ence and persistence, blood pressure, and lowdensity lipoprotein cholesterol. JAMA. 2006; 296: 2563.

6. Brown MT, Bussell JK. Medication adherence: WHO cares? Mayo Clin Proc. 2011; 86: 304-14.

7. Lam WY, Fresco P. Medication adherence measures: an overview. BioMed Res Int. 2015; 2015: 217047.

8. Velligan DI, Wang M, Diamond P, et al. Relationships among subjective and objective measures of adherence to oral antipsychotic medications. Psychiatr Serv. 2007; 58: 1187-92.

9. Lavsa SM, Holzworth A, Ansani NT. Selection of a validated scale for measuring medication adherence. J Am Pharm Assoc. 2011; 51: 90-4.

10. Nguyen T-M-U, Caze A La, Cottrell N. What are validated self-report adherence scales really measuring?: a systematic review. Br J Clin Pharmacol. 2014; 77: 427-45.

11. Sanders EB-N, Stappers PJ. Co-creation and the new landscapes of design. CoDesign. 2008; 4: 5-18.

12. Luxford K, Safran DG, Delbanco T. Promoting patient-centered care: a qualitative study of facilitators and barriers in healthcare organizations with a reputation for improving the patient experience. Int J Qual Heal Care. 2011; 23: $510-5$

13. Doyle C, Lennox L, Bell D. A systematic review of evidence on the links between patient experience and clinical safety and effectiveness. BMJ Open. 2013; 3.

14. Slattery P, Saeri AK, Bragge P. Research codesign in health: a rapid overview of reviews. Heal Res Policy Syst. 2020; 18: 17.

15. Anghel L-A, Farcaş AM, Oprean RN. Medication adherence and persistence in patients with autoimmune rheumatic diseases: A narrative review. Patient Prefer Adherence. 2018; 12: 1151-66.

16. Costedoat-Chalumeau N, Pouchot J, GuettrotImbert G, et al. Adherence to treatment in systemic lupus erythematosus patients. Best Pract Res Clin Rheumatol. 2013; 27: 329-40.

17. Liberati A, Altman DG, Tetzlaff J, et al. The PRISMA statement for reporting systematic reviews and meta-analyses of studies that evaluate health care interventions: explanation and elaboration. PLoS Med. 2009; 6: e1000100.

18. Du X, Chen H, Zhuang Y, et al. Medication adherence in chinese patients with systemic lupus erythematosus. J Clin Rheumatol. 2020; 26: 94-8.

19. Hachulla E, Gouellec N Le, Launay D, et al. Adherence to hydroxychloroquine in patients with systemic lupus: contrasting results and weak correlation between assessment tools. J Bone Spine. 2020 [Epub ahead of print].

20. Harry O, Crosby LE, Mara C, et al. Feasibility 
and acceptability of an innovative adherence intervention for young adults with childhoodonset systemic lupus erythematosus. Pediatr Rheumatol. 2020; 18: 36.

21. Ali AY, Abdelaziz TS, Essameldin M. The prevalence and causes non-adherence to immunosuppressive medications in patients with Lupus nephritis flares. Curr Rheumatol Rev. 2019; 15.

22. Brijs J, Arat S, Westhovens R, et al. Treatment adherence in systemic sclerosis: a cross-sectional study. Musculoskeletal Care. 2019; 17: 44-53.

23. Clowse MEB, Eudy AM, Revels J, et al. Provider perceptions on the management of lupus during pregnancy: barriers to improved care. Lupus. 2019; 28: 86-93.

24. Amalia R, Sasongko H, Kundarto W, Niruri R. The analysis of the factors affecting medication adherence in patient with SLE (systemic lupus erythematosus) at Yayasan Tittari Griya Kupu solo. Indian J Public Heal Res Dev. 2019; 10: 672-6.

25. Sun K, Eudy AM, Rogers JL, et al. 197 Racial disparities in lupus medication adherence. Lupus Sci Med. 2019; 6: A147-8.

26. Wallace DJ, Tse K, Hanrahan L, et al. Hydroxychloroquine usage in US patients, their experiences of tolerability and adherence, and implications for treatment: Survey results from 3127 patients with SLE conducted by the Lupus Foundation of America. Lupus Sci Med. 2019; 6(1).

27. Zayed HS, Medhat BM, Seif EM. Evaluation of treatment adherence in patients with $\mathrm{Be}$ hçet's disease: its relation to disease manifestations, patients' beliefs about medications, and quality of life. Clin Rheumatol. 2019; 38: 761-8.

28. Khabbazi A, Karkon Shayan F, Ghojazadeh $\mathrm{M}$, et al. Adherence to treatment in patients with Behçet's disease. Int J Rheum Dis. 2018; 21: 2158-66.

29. Georgopoulou S, Nel L, Sangle S, et al. O20 Physician-patient interaction and medication adherence in lupus nephritis. Rheumatology. 2018; 57: iii32-3.

30. Costedoat-Chalumeau N, Houssiau F, Izmirly $\mathrm{P}$, et al. A prospective international study on adherence to treatment in 305 patients with flaring SLE: assessment by drug levels and self-administered questionnaires. Clin Pharmacol Ther. 2018; 103: 1074-82.

31. Iudici M, Pantano I, Fasano S, et al. Health status and concomitant prescription of immunosuppressants are risk factors for hydroxychloroquine non-adherence in systemic lupus patients with prolonged inactive disease. Lupus. 2018; 27: 265-72.

32. Alsowaida N, Alrasheed M, Mayet A, Alsuwaida A, Omair MA. Medication adherence, depression and disease activity among patients with systemic lupus erythematosus. Lupus. 2018; 27: 327-32.

33. Johnsen K, Jolly M, Annapureddy N. Health related quality of life in lupus: self-management-a modifiable predictor. Arthritis Rheumatol. 2017; 69: 1-3.

34. Perel-Winkler A, Neville K, Nguyen S, et al. Low health literacy does not impact adherence to hydroxychloroquine in patients with systemic lupus. Arthritis Rheumatol. 2017; 69.

35. Farinha F, Freitas F, Águeda A, et al. Concerns of patients with systemic lupus erythematosus and adherence to therapy - a qualitative study. Patient Prefer Adherence. 2017; 11: 1213-9.

36. Çinar M, Tekgöz E, Çınar Fi, Yılmaz S. Reliability and validity of the turkish translation of the beliefs about medicines questionnaire (BMQ-T) in patients with Behçet's disease. Turkish Thorac J. 2018; 19: 231-2.

37. Flower C, Hambleton I, Campbell M. The effect of psychosocial and neuropsychiatric factors on medication adherence in a cohort of women with systemic lupus erythematosus. J Clin Rheumatol. 2016; 22: 411-7.

38. Drenkard C, Mathew S, Bao G, Lim SS. Depression Is a Risk Factor for Low Treatment Adherence in African American People with Systemic Lupus Erythematosus. Arthritis Rheumatol. 2016; 68 (suppl 10).

39. Singh JA, Qu H, Yazdany J, et al. Barriers to medication decision making in women with lupus nephritis: a formative study using nominal group technique. J Rheumatol. 2015; 42: 1616-23.

40. Hale ED, Radvanski DC, Hassett AL. The man-in-the-moon face: a qualitative study of body image, self-image and medication use in systemic lupus erythematosus. Rheumatology (Oxford). 2015; 54: 1220-5.

41. Hromadkova L, Soukup T, Cermakova E, V1cek J. Drug compliance in patients with systemic scleroderma. Clin Rheumatol. 2012; 31: 1577-83.

42. Daleboudt GMN, Broadbent E, McQueen F, Kaptein AA. Intentional and unintentional treatment nonadherence in patients with systemic lupus erythematosus. Arthritis Care Res. 2011; 63: 342-50.

43. Duvdevany I, Cohen M, Minsker-Valtzer A, Lorber M. Psychological correlates of adherence to self-care, disease activity and functioning in persons with systemic lupus erythematosus. Lupus. 2011; 20: 14-22.

44. Bennett JK, Fuertes JN, Keitel M, Phillips R. The role of patient attachment and working alliance on patient adherence, satisfaction, and health-related quality of life in lupus treatment. Patient Educ Couns. 2011; 85: 53-9.

45. Chambers SA, Raine R, Rahman A, Isenberg D. Why do patients with systemic lupus erythematosus take or fail to take their pre- 
scribed medications? A qualitative study in a UK cohort. Rheumatology (Oxford). 2009; 48: 266-71.

46. Chambers S, Raine R, Rahman A, et al. Factors influencing adherence to medications in a group of patients with systemic lupus erythematosus in Jamaica. Lupus. 2008; 17: 761-9.

47. Koneru S, Kocharla L, Higgins GC, et al. Adherence to medications in systemic lupus erythematosus. J Clin Rheumatol. 2008; 14: 195-201.

48. Garcia Popa-Lisseanu MG, Greisinger A, Richardson $\mathrm{M}$, et al. Determinants of treatment adherence in ethnically diverse, economically disadvantaged patients with rheumatic disease. J Rheumatol. 2005; 32: 913-9.

49. Vitolins MZ, Rand CS, Rapp SR, et al. Measuring adherence to behavioral and medical interventions. Control Clin Trials. 2000; 21: 188S-94S.

50. Garfield S, Clifford S, Eliasson L, et al. Suitability of measures of self-reported medication adherence for routine clinical use: a systematic review. BMC Med Res Methodol. 2011; 11: 149.

51. Walsh JC, Mandalia S, Gazzard BG. Responses to a 1 month self-report on adherence to antiretroviral therapy are consistent with electronic data and virological treatment outcome. AIDS. 2002; 16: 269-77.

52. Morisky DE, Green LW, Levine DM. Concurrent and predictive validity of a self-reported measure of medication adherence. Med Care. 1986; 24: 67-74.

53. Morisky DE, Ang A, Krousel-Wood M, Ward HJ. Predictive validity of a medication adherence measure in an outpatient setting. J Clin Hypertens. 2008; 10: 348-54.

54. Svarstad BL, Chewning BA, Sleath BL, Claesson $\mathrm{C}$. The brief medication questionnaire: a tool for screening patient adherence and barriers to adherence. Patient Educ Couns. 1999; 37: 113-24.

55. Farmer KC. Methods for measuring and monitoring medication regimen adherence in clini- cal trials and clinical practice. Clin Ther. 1999; 21: 1074-90; discussion 1073.

56. Tan X, Patel I, Chang J. Review of the four item Morisky Medication Adherence Scale (MMAS-4) and eight item Morisky Medication Adherence Scale (MMAS-8). Inov Pharm. 2014; 5(3).

57. Morisky DE, Ang A, Krousel-Wood M, Ward HJ. Predictive validity of a medication adherence measure in an outpatient setting. J Clin Hypertens. 2008; 10: 348-54.

58. Koneru S, Shishov M, Ware A, et al. Effectively measuring adherence to medications for systemic lupus erythematosus in a clinical setting. Arthritis Rheum. 2007; 57: 1000-6.

59. de Klerk E, van der Heijde D, Landewé R, et al. The compliance-questionnaire-rheumatology compared with electronic medication event monitoring: a validation study. J Rheumatol. 2003; 30: 2469-75.

60. Hughes LD, Done J, Young A. A 5 item version of the Compliance Questionnaire for Rheumatology (CQR5) successfully identifies low adherence to DMARDs. BMC Musculoskelet Disord. 2013; 14: 286.

61. Náfrádi L, Nakamoto K, Schulz PJ. Is patient empowerment the key to promote adherence? A systematic review of the relationship between self-efficacy, health locus of control and medication adherence. PLoS One. 2017; 12 (10).

62. van den Bemt BJF, Gettings L, Domańska B, et al. A portfolio of biologic self-injection devices in rheumatology: how patient involvement in device design can improve treatment experience. Drug Deliv. 2019; 26: 384-92.

63. Chow S-C, Chang Y-W. Statistical considerations for rare diseases drug development. J Biopharm Stat. 2019; 29: 874-86.

64. Jessup RL, Osborne RH, Buchbinder R, Beauchamp A. Using co-design to develop interventions to address health literacy needs in a hospitalised population. BMC Health Serv Res. 2018; 18: 989. 